\title{
Feature-based non-parametric estimation of Kullback- Leibler divergence for SAR image change detection
}

\author{
Shiyong Cui $\mathbb{1}^{\mathrm{a}}$ and Chengfeng Luo $\mathbb{1}^{\mathrm{b}}$
}

aRemote Sensing Technology Institute (IMF), German Aerospace Center (DLR), Wessling, Germany; ${ }^{\text {blnstitute of }}$ Photogrammetry and Remote Sensing, Chinese Academy of Surveying and Mapping (CASM), Beijing, China

\begin{abstract}
In this article, a method based on a non-parametric estimation of the Kullback-Leibler divergence using a local feature space is proposed for synthetic aperture radar (SAR) image change detection. First, local features based on a set of Gabor filters are extracted from both preand post-event images. The distribution of these local features from a local neighbourhood is considered as a statistical representation of the local image information. The Kullback-Leibler divergence as a probabilistic distance is used for measuring the similarity of the two distributions. Nevertheless, it is not trivial to estimate the distribution of a high-dimensional random vector, let alone the comparison of two distributions. Thus, a non-parametric method based on $k$-nearest neighbour search is proposed to compute the Kullback-Leibler divergence between the two distributions. Through experiments, this method is compared with other state-of-the-art methods and the effectiveness of the proposed method for SAR image change detection is demonstrated.
\end{abstract}

ARTICLE HISTORY

Received 8 May 2016 Accepted 29 June 2016

\section{Introduction}

Due to the all-weather operating ability of synthetic aperture radar (SAR), high-resolution SAR images acquired by modern spaceborne systems, such as TerraSAR-X and TanDEM-X, (Wikipedia 2016) are playing an important role in various applications such as environmental monitoring, disaster assessment and land cover dynamics. A typical requirement in these applications is change detection and a large number of different methods have been proposed in this context. In the following, we briefly review related methods.

In general, most change detection methods consists of three steps: preprocessing, image comparison to generate a change map and change map analysis to generate a final binary change map. Preprocessing includes image registration, radiometric corrections, despeckling, etc. A widely used image comparison technique for SAR images is the log-ratio operator (Rignot and Van Zyl 1993; Bazi, Bruzzone, and Melgani 2005; Celik 2010), which is particularly suited to SAR change detection due to the presence of multiplicative noise. A method based on a likelihood ratio between the statistical distributions of SAR intensity images was proposed for SAR image change detection by Xiong, Chen, and Kuang (2012). Information 
measures have been applied to change detection as well and have shown promising performances. A prominent work by Inglada and Mercier (2007) proposed a method for multi-temporal SAR change detection based on the evolution of local statistics computed from the pre-event and post-event images. The local statistics are estimated by one-dimensional Edgeworth series expansion, which approximates the probability density functions of the pixels in the neighbourhood. The degree of evolution of the local statistics is measured using the Kullback-Leibler divergence. Bovolo and Bruz-zone (2008) extended this method to object-based change detection by computing the Kullback-Leibler divergence of two corresponding regions obtained by image segmentation. An unsupervised change detection method in the wavelet domain based on statistical wavelet coefficient modelling was proposed by Cui and Datcu (2012). Several information similarity measures, namely distance to independence, mutual information, cluster reward, Woods criterion and correlation ratio, were compared by Alberga (2009) for change detection, among which mutual information has been demonstrated to be rather efficient. Taking advantage of mutual information, a pixel-based approach comparing localized mutual information was proposed by Winter et al. (1997). Intuitively, if two pixels share a lot of information, it is reasonable to assume no change at their location. Based on this idea, another information measure for change detection derived from mutual information was introduced by Gueguen and Datcu (2009), namely mixed information, which unifies mutual information and variational information by a parameter. Furthermore, stochastic kernels including both Kullback-Leibler divergence and mutual information were used by Mercier et al. (2006) as features in a support vector machine for SAR change detection. Based on the estimation of a bivariate Gamma distribution, mutual information was applied to SAR change detection by Chatelain et al. (2007). Through a twoscale implementation, mutual information can be split into two terms to be linked to a change detection part and a registration part (Mercier and Inglada 2008). The method by Bovolo and Bruzzone (2005) exploits a wavelet-based multiscale decomposition of the log-ratio image aiming at representation of the change signal using different scales. $k$-means clustering was applied by Celik (2009) to classify the undecimated wavelet transform of the difference image into two classes corresponding to change and no-change classes. A benchmark evaluation of similarity measures, namely mutual information, variational information, mixed information and Kullback-Leibler divergence, was carried out by Cui, Schwarz, and Datcu (2016) for multitemporal SAR image change detection.

Most works reviewed earlier perform change detection using pixel intensities, i.e., local pixel statistics are computed and used. However, features, such as texture features, are rarely used for change detection since it is not really trivial in practice to estimate the distribution of a high-dimensional random vector, let alone the comparison of two distributions. An unsupervised SAR change detection method using Gabor features based on two-level clustering was proposed by Li et al. (2015). However, this method avoids the problem of high dimensionality by clustering. Motivated by this observation, a feature-based non-parametric method is proposed in this article. The contributions of this letters are twofold. First, in contrast to most intensity-based methods, this is a new feature-based method for change detection. Second, the problem in computing the Kullback-Leibler divergence between two distributions of high-dimensional random vectors is solved by $k$-nearest neighbour $(k-N N)$ search. An obvious advantage of this solution is that one does not have to assume any functional form for the underlying distribution. It has mainly two steps. First, a set of Gabor filters is used to extract local features from a local neighbourhood that are deemed as a representation of the local 
information. Then the distributions of the feature spaces are compared for assessing the image similarity by the Kullback-Leibler divergence. To avoid estimating the distribution of the local features and to reduce the complexity in computing the Kullback-Leibler divergence, a non-parametric method based on $k$-NN search is proposed.

In the following sections, random variables are denoted by $X$ and $Y$. Local feature vectors are represented by $\boldsymbol{x}_{i}$ and $\boldsymbol{y}_{i}$. The set of local features are denoted by X and Y. $d$ is the number of dimensions in the local feature vector.

\section{Local Gabor feature extraction}

Gabor features have been widely used in various applications for texture characterization. Various texture features based on Gabor filters have been compared by Grigorescu, Petkov, and Kruizinga (2002). In the context of SAR image analysis, Gabor filters have been applied in many works as well, such as the texture analysis performed by Kandaswamy, Adjeroh, and Lee (2005). Gabor texture features have been used by Dumitru et al. (2015) for large-scale SAR image annotation. Generally, Gabor filter is a kind of wavelet transform, which has shown good performance in texture discrimination. Gabor functions form a complete but non-orthogonal basis set. Expanding a signal using this basis provides a localized frequency description. A two-dimensional Gabor function $g(x, y)$, with $x$ and $y$ being pixel coordinates, is defined as

$$
g(x, y)=\left(\frac{1}{2 \pi \sigma_{x} \sigma_{y}}\right) \exp \left[-\frac{1}{2} \frac{x^{2}}{\sigma_{x}^{2}}+\frac{y^{2}}{\sigma_{y}^{2}}+2 \pi j W x\right],
$$

where $W$ is the central frequency and $\sigma_{x}$ and $\sigma_{y}$ are the standard deviations of $x$ and $y$, respectively. This is actually the product of a Gaussian function with a complex sinusoid. Its Fourier transform (Manjunath and Ma 1996) are defined as

$$
G(u, v)=\exp \left\{-\frac{1}{2}\left[\frac{(u-W)^{2}}{\sigma_{u}^{2}}+\frac{v^{2}}{\sigma_{v}^{2}}\right]\right\},
$$

where $\sigma_{u}=1 / 2 \pi \sigma_{x}, \sigma_{v}=1 / 2 \pi \sigma_{y}$ and $u$ and $v$ are the frequency coordinates. This forms a bandpass filter in the frequency domain, where the bandwidth and the centre frequency of the filter are controlled by the standard deviation of the Gaussian function and the frequency of the complex sinusoid, respectively.

Then, a Gabor filter bank can be obtained using appropriate dilations $a$ and rotations $\theta$ through the following generating function:

$$
\begin{aligned}
& g_{m, n}(x, y)=a^{-m} g\left(x^{\prime}, y^{\prime}\right), \quad a>1, \quad m, n \in \mathbb{Z} \\
& x^{\prime}=a^{-m}(x \cos \theta+y \sin \theta), \\
& y^{\prime}=a^{-m}(-x \sin \theta+y \cos \theta),
\end{aligned}
$$

where $\theta=n \pi / k$ and $k$ is the number of orientations and $m$ is the number of scales. They are two important parameters that have to be well selected when extracting Gabor feature. After applying Gabor filter bank to both images, the local statistics of the filter responses, i.e., mean and standard deviation, are calculated and used as feature vectors. Thus, each pixel has a feature vector that is considered as a representation of the local context. 


\section{Non-parametric estimation of Kullback-Leibler divergence}

In order to quantify the degree of change between two images, the Kullback-Leibler divergence is applied at each pixel based on two corresponding distributions of the local Gabor features.

\subsection{Kullback-Leibler divergence}

Suppose $p_{X}(x)$ and $p_{Y}(x)$ are probability distributions of the local feature $\boldsymbol{x}$ taking values from $\mathbb{R}^{d}$, the Kullback-Leibler divergence between them is defined as

$$
D(X \| Y)=\int p_{X}(x) \ln \frac{p_{X}(x)}{p_{Y}(x)} \mathrm{d} x
$$

If the two probability density functions $p_{X}(x)$ and $p_{Y}(x)$ are close to each other, the Kullback-Leibler divergence is small. In contrast, it is larger if there is a great deviation between them. In order to compute it, the two density distributions $p_{X}(x)$ and $p_{Y}(x)$ have to be estimated using the local features. However, density estimation is always a hard problem in practice except particular cases, like SAR image modelling, where a strong prior for the functional form of the distributions is available. In these cases, the two distributions are parametric distributions that can be obtained by estimating the governing parameters. Then the Kullback-Leibler divergence can be computed by substituting the two parametric distributions in Equation (4). Even so, the KullbackLeibler divergence might still be analytically intractable in some cases. If no prior information about the distributions is available, one has to resort to nonparametric estimation approach. The traditional non-parametric approach is to use a histogram with equally sized bins to estimate the densities $p_{X}(x)$ and $p_{Y}(x)$ and substitute the density estimates $\hat{p}_{X}(x)$ and $\hat{p}_{Y}(x)$ in Equation (4). However, this method has a number of drawbacks, for instance, starting point of the bins and bin sizes. Furthermore, the histogram estimate is not continuous. A much more serious problem is the curse of dimensionality, since the number of bins grows exponentially with the number of dimensions $d$. From this point of view, $k$-NN density estimation is a better choice.

\section{2. $k-N N$ divergence estimator}

In the case of no prior information, non-parametric estimation of the Kullback-Leibler divergence is much more preferable to parametric approaches because one does not have to assume any functional form of the underlying distribution. A universal non-parametric estimator of divergence was proposed by Wang, Kulkarni, and Verdu (2009) for multidimensional continuous densities based on $k$-NN distances. It has been demonstrated to be asymptotically unbiased and mean-square consistent for independent and identically distributed (IID) samples. Suppose $X=\left\{\boldsymbol{x}_{1}, \boldsymbol{x}_{2}, \cdots, \boldsymbol{x}_{N}\right\}$ and $Y=\left\{\boldsymbol{y}_{1}, \boldsymbol{y}_{2}, \cdots, \boldsymbol{y}_{M}\right\}$ are local feature vectors from two corresponding windows of the two images and let $\rho_{k}\left(x_{i}\right)$ be the Euclidean distance between $\boldsymbol{x}_{i}$ and its $k$ th neighbour in $X$ and $v_{k}\left(x_{i}\right)$ be the Euclidean distance between $x_{i}$ and its $k$ th neighbour in $Y$. The samples in $X$ and $Y$ are considered as $d$-dimensional IID samples drawn from $p_{X}(x)$ and $p_{Y}(x)$. Based on the basic principle of non-parametric density estimation, the general expression of the density at $\boldsymbol{x}$ is 


$$
\hat{p}_{X}(x)=\frac{k}{N V}
$$

where $V$ is a volume surrounding $\boldsymbol{x}, N$ is the total number of examples and $k$ is the number of examples inside $V$. In applying this principle to practical density estimation problems, there are two basic approaches that one can choose. One can use a fixed value of $k$ and determine the corresponding volume $V$ from the data, which leads to the $k$-NN approach. Alternatively, one can employ a fixed value of the volume $V$ and then determine the number of samples $k$ from the data, leading to kernel density estimation. The method proposed by Wang, Kulkarni, and Verdu (2009) falls into the $k$-NN category, where a volume surrounding the estimation point $\boldsymbol{x}$ and enclosing a total of $k$ points is considered. The $d$-dimensional volume of a Euclidean ball of radius $R$ in $n$-dimensional Euclidean space is

$$
V_{d}(R)=V_{1}(d) R^{d}=\frac{\pi^{d / 2}}{\Gamma\left(\frac{d}{2}+1\right)} R^{d}
$$

where $V_{1}(d)$ is the volume of the unit ball in $\mathbb{R}^{d}$ and $\Gamma$ is the Gamma function. Substituting Equation (6) in Equation (5), one can obtain the $k$-NN density estimate of $p_{X}\left(x_{i}\right)$ at $x_{i}$

$$
\hat{p}_{X}\left(x_{i}\right)=\frac{k}{N-1} \frac{1}{V_{1}(d) \rho_{k}^{d}\left(x_{i}\right)}
$$

Similarly, the $k$-NN density estimate of $p_{Y}\left(x_{i}\right)$ at $x_{i}$ is

$$
\hat{p}_{Y}\left(x_{i}\right)=\frac{k}{M} \frac{1}{V_{1}(d) v_{k}^{d}\left(x_{i}\right)}
$$

Based on the law of large numbers, the Kullback-Leibler divergence can be approximated as

$$
D(X \| Y)=\int p_{X}(x) \ln \frac{p_{X}(x)}{p_{Y}(x)} d x \approx \frac{1}{N} \sum_{i=1}^{N} \ln \frac{\hat{p}_{X}\left(x_{i}\right)}{\hat{p}_{Y}\left(x_{i}\right)}
$$

Substituting Equations (7) and (8) in Equation (9), one can obtain the universal estimate of the Kullback-Leibler divergence in Equation (10).

$$
\hat{D}(X \| Y)=\frac{d}{N} \sum_{i=1}^{N} \ln \frac{v_{k}\left(x_{i}\right)}{\rho_{k}\left(x_{i}\right)}+\ln \frac{M}{N-1}
$$

Due to its asymmetry, the symmetric version of Kullback-Leibler divergence $D(X, Y)=$ $\hat{D}(X \| Y) / 2+\hat{D}(Y \| X) / 2$ is used for SAR image change detection in this article. Since the distance $\rho_{k}\left(x_{i}\right)$ and $v_{k}\left(x_{i}\right)$ of a sample $x_{i}$ to its $k$ th neighbour can be computed fast using some useful data structure such as $k$-dimensional tree (Wald and Havran 2006) and locality sensitive hashing (Slaney and Casey 2008), the Kullback-Leibler divergence can be evaluated quickly. 


\section{Experiments and discussions}

\subsection{Datasets}

To evaluate the proposed method for SAR image change detection, a subset with a size of $613 \times 641$ pixels, shown in Figure 1, was selected from two radiometrically enhanced TerraSAR-X images acquired in Stripmap mode prior to (on 20 October 2010) and after (on 6 May 2011) the Sendai earthquake in Japan. Their pixel spacing is about $2.5 \mathrm{~m}$. The reference data shown in Figure 1 (c) was produced through careful manual interpretation by referring optical images. Due to the earthquake, a tsunami occurred, which led to a devastating flooding, as can be seen from the images.

\subsection{Experimental set-up}

To evaluate the quality of the change map independent of any thresholding algorithm, the receiver operating characteristic (ROC) curve is used and the area under ROC curve (AUC) is computed as a performance measure. ROC curve can be considered as the evolution of true positive rate (TPR) as a function of false alarm rate (FAR). The area under ROC curve is a good performance measure. The larger the area under ROC curve, the better the performance. As AUC is an overall performance measure, thresholding or labelling of the change index should also be applied to generate a binary changedetection map, such that TPR and FAR can be used as well for evaluation and comparison. Although many thresholding methods have been proposed, none of them is perfect. Therefore, an optimal threshold corresponding to the nearest point to $(0.0$, 1.0) on the ROC curve is selected, which gives the best performance. Different windows sizes $(5 \times 5,7 \times 7,9 \times 9,11 \times 11,13 \times 13,15 \times 15,17 \times 17,19 \times 19,21 \times 21$ and $23 \times$ 23 pixels) are used. The number of scales and orientation in Gabor filter bank are set to 4 and 6 . The parameter $k$ in $k$-NN is set to 3 . The methods proposed by Inglada and Mercier (2007) and Li et al. (2015) were selected for the purpose of comparison, which is referred as KL_EW and GabTLC in the following. Since GabTLC directly generates a binary change map based on clustering, it is not possible to compute AUC but only TPR and FAR. The same experimental set-up was used. The method propose in this article is referred as KL_NP in the following sections.

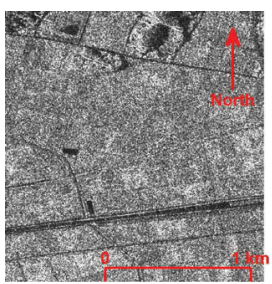

(a)

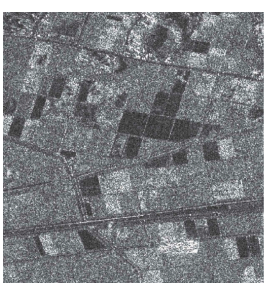

(b)



(c)

Figure 1. Test data: (a) TerraSAR-X image acquired before earthquake in Japan on October 20, 2010; (b) TerraSAR-X image acquired after earthquake in Japan on May 6, 2011); (c) ground reference data. The location (centre) of the image is at $38^{\circ} 8^{\prime} 47.43^{\prime \prime} \mathrm{N}, 140^{\circ} 52^{\prime} 7.36^{\prime \prime} \mathrm{E}$. The white pixels are the changed sites and the black pixels are the places without change. 


\subsection{Results and discussions}

The AUCs of the two methods, i.e., KL_EW and KL_NP, are shown in the first two rows in Table 1 and are plotted in Figure 2(a). It is clear that KL_NP performs much better than KL_EW for windows larger than $9 \times 9$ pixels. In the best case with a window size of $23 \times 23$, the AUC of KL_NP is $8.7 \%$ better than that of KL_EW. The AUC of KL_NP increases steadily as the window size increases and becomes stable beyond a window size of $21 \times 21$ pixels. In contrast, KL_EW achieves the maximum accuracy at $9 \times 9$ and decreases significantly beyond that size. The reason is that $\mathrm{KL}$ EW relies on one-dimension Edgeworth series expansion, thus less samples are needed for a sufficient estimation. However, the dimension of the feature vectors used in KL_NP is much larger than that of KL_EW. Thus, it requires more samples for an accurate estimation, which means a large window size.

In addition to AUC, TPR and FAR are shown in Table 1 as well. Accordingly, for KL_EW and KL_NP, TPR follows a similar behaviour, as can be seen from Figure 2(b). TPR of KL_NP grows firmly as the window size increases and converges at the size of $21 \times 21$. In contrast, TPR of KL_EW increases slightly from window size of $5 \times 5$ till $9 \times 9$ and after that it declines steadily as the window size increases. It is striking that the TPR of GabTLC is much higher than KL_EW and KL_NP for window sizes smaller than $21 \times 21$ pixels but lower for larger window sizes. The behaviour of FAR for the three methods is shown in Figure 2(c). For KL_NP, FAR drops firmly as the window size increases. However, FAR of KL_EW increases steadily with increasing window size. As confirmations, ROC curves of the two methods using window sizes of $7 \times 7,15 \times 15$ and $23 \times 23$ are shown in Figure 3 . One can see that AUC of KL_NP is smaller than KL_EW for a smaller window size such as $5 \times 5$ pixels. But for a larger window size, AUC of KL_NP is significantly larger than that of KL_EW. Although GabTLC has a much higher TPR, it has a

Table 1. Comparison of the two methods in terms of AUC, TPR and FAR using different window sizes.

\begin{tabular}{llccccccccccc}
\hline Accuracy measure & Method & \multicolumn{10}{c}{ Window size (pixels) } \\
\hline \multirow{3}{*}{ AUC } & & 5 & 7 & 9 & 11 & 13 & 15 & 17 & 19 & 21 & 23 \\
& KL EW & 92.90 & 94.09 & 94.21 & 93.91 & 93.34 & 92.62 & 91.87 & 91.10 & 90.32 & 89.54 \\
\multirow{4}{*}{ TPR } & KL NP & 88.69 & 91.91 & 94.21 & 95.81 & 96.87 & 97.52 & 97.90 & 98.11 & 98.22 & 98.26 \\
& GabTLC & - & - & - & - & - & - & - & - & - & - \\
& KL EW & 86.53 & 87.77 & 87.84 & 87.78 & 86.52 & 86.65 & 85.73 & 84.44 & 83.80 & 82.71 \\
FAR & KL NP & 80.18 & 83.43 & 86.48 & 88.35 & 89.86 & 91.17 & 92.00 & 93.37 & 92.75 & 92.77 \\
& GabTLC & 88.29 & 91.79 & 97.07 & 98.70 & 99.06 & 99.01 & 98.56 & 96.33 & 81.43 & 77.25 \\
& KL EW & 14.83 & 14.39 & 14.55 & 15.14 & 14.69 & 16.54 & 17.30 & 18.01 & 19.17 & 19.80 \\
& KL NP & 19.27 & 15.75 & 13.21 & 11.25 & 09.30 & 08.24 & 07.42 & 07.71 & 06.80 & 06.82 \\
& GabTLC & 16.42 & 12.06 & 17.77 & 16.60 & 16.09 & 16.38 & 17.36 & 18.93 & 13.17 & 21.02 \\
\hline
\end{tabular}

The best accuracy for each method is marked by bold font.

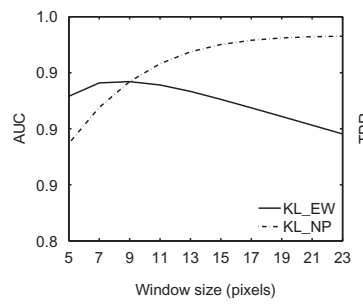

(a)

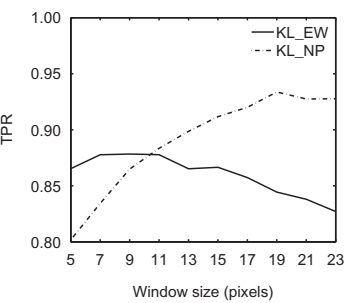

(b)

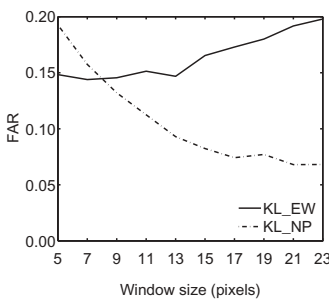

(c)

Figure 2. Comparison of KL_EW and KL_NP using various window sizes: (a) AUCs of the two methods; (b) TPRs of the two methods; (c) FARs of the two methods. 


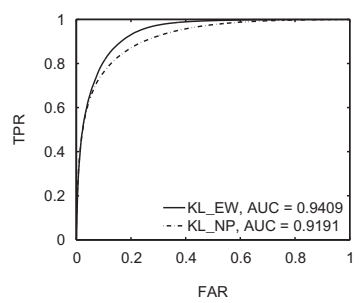

(a)

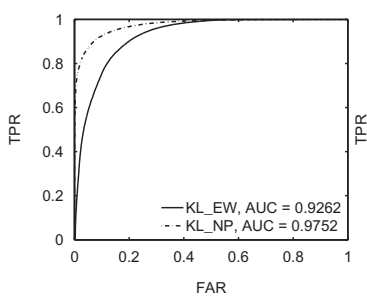

(b)

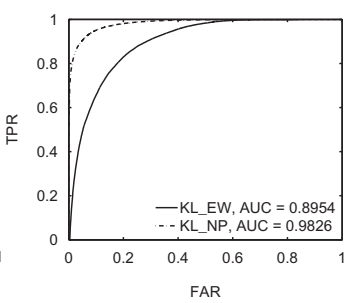

(c)

Figure 3. ROC curves of KL_EW and KL_NP using different window sizes: (a) ROC curves of the two methods using a window size of $7 \times 7$ pixels; $(b)$ ROC curves of the two methods using a window size of $15 \times 15$ pixels; (c) ROC curves of the two methods using a window size of $23 \times 23$ pixels.

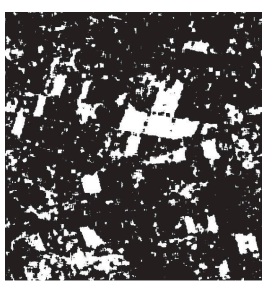

(a)

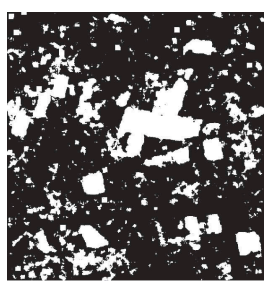

(b)

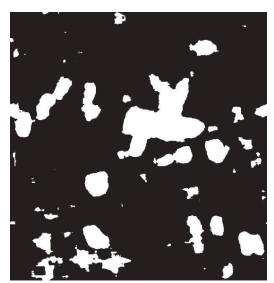

(c)

Figure 4. Best change maps of the study area: (a) change map of KL_EW using a window size of $9 \times 9$ pixels; (b) change map of GabTLC using a window size of $13 \times 13$ pixels; (c) change map of $\mathrm{KL} \_N P$ using a window size of $23 \times 23$ pixels.

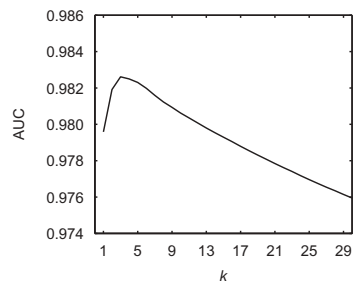

(a)

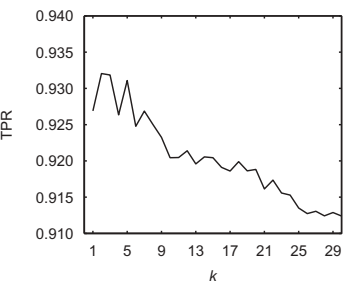

(b)

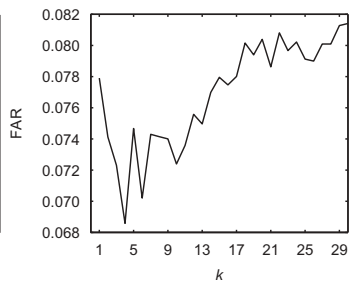

(c)

Figure 5. Influence of the parameter $k$ in $k$-NN on the performance: $(a)$ influence of $k$ on AUC; $(b)$ influence of $k$ on TPR; $(c)$ influence of $k$ on FAR.

much higher FAR as well that can be confirmed from the change map shown in Figure 4(b). The parameter $k$ used in the $k$-NN is an important parameter. Its influence on the performance is shown in Figure 5 . One can observe that the method performs best when $k=3$. Another set of parameters is the number of scales and orientations used in the Gabor filter ban Their influences on the performance are shown in Table 2. One can see that the performance is not sensitive to these two parameters.

\section{Conclusion}

In this article, a method based on non-parametric estimation of the Kullback-Leibler divergence for SAR image change detection is proposed and evaluated. First a set of 
Table 2. The influence of the parameters in Gabor filter on the performance in terms of AUC, TPR and FAR.

\begin{tabular}{lcccccccc}
\hline Accuracy measure & \multicolumn{1}{l}{ Scale } & \multicolumn{7}{c}{ Orientation } \\
\hline \multirow{3}{*}{ AUC } & & 2 & 3 & 4 & 5 & 6 & 7 & 8 \\
& 2 & 98.10 & 98.26 & 98.48 & 98.42 & 98.39 & 98.32 & 98.28 \\
& 3 & 98.29 & 98.32 & 98.18 & 98.13 & 98.16 & 98.15 & 98.17 \\
& 4 & 98.47 & 98.21 & 98.14 & 98.14 & 98.26 & 98.29 & 98.35 \\
TPR & 5 & 98.34 & 98.18 & 98.10 & 98.20 & 98.35 & 98.40 & 98.45 \\
& 2 & 92.97 & 93.27 & 93.89 & 93.56 & 93.76 & 93.50 & 93.56 \\
& 3 & 93.22 & 93.25 & 92.77 & 92.89 & 92.74 & 92.41 & 92.74 \\
FAR & 4 & 94.29 & 92.98 & 92.88 & 93.26 & 93.18 & 93.39 & 93.29 \\
& 5 & 93.10 & 92.90 & 92.95 & 93.30 & 93.23 & 93.55 & 93.63 \\
& 2 & 7.03 & 6.78 & 6.61 & 6.66 & 7.13 & 7.22 & 7.67 \\
& 3 & 6.97 & 7.06 & 6.96 & 7.69 & 7.26 & 7.13 & 7.50 \\
& 4 & 7.10 & 7.11 & 7.18 & 7.90 & 7.23 & 7.16 & 7.03 \\
\hline
\end{tabular}

Gabor filters is used to extract local features, which forms a local feature space. The Kullback-Leibler divergence as a probabilistic distance is used for measuring the similarity of the two distributions. Due to the curse of dimensionality, it is not really trivial in practice to estimate the distribution of a high-dimensional random vector, let alone the comparison of two distributions. To avoid any parametric assumption of the distribution and reduce the complexity of in computation, the Kullback-Leibler divergence is estimated based on $k$-NN. This method is compared with other state-of-the-art method and the effectiveness of the proposed method in SAR image change detection is demonstrated.

\section{Disclosure statement}

No potential conflict of interest was reported by the authors.

\section{ORCID}

Shiyong Cui (iD) http://orcid.org/0000-0002-5417-4482

Chengfeng Luo (10) http://orcid.org/0000-0002-5857-2226

\section{References}

Alberga, V. 2009. "Similarity Measures of Remotely Sensed Multi-sensor Images for Change Detection Applications." Remote Sensing 1 (3): 122-143. doi:10.3390/rs1030122.

Bazi, Y., L. Bruzzone, and F. Melgani. 2005. "An Unsupervised Approach Based on the Generalized Gaussian Model to Automatic Change Detection in Multitemporal SAR Images." IEEE Transactions on Geoscience and Remote Sensing 43 (4, April): 874-887. doi:10.1109/TGRS.2004.842441.

Bovolo, F., and L. Bruzzone. 2005. "A Detail-Preserving Scale-Driven Approach to Change Detection in Multitemporal SAR Images." IEEE Transactions on Geoscience and Remote Sensing 43 (12): 2963-2972. doi:10.1109/TGRS.2005.857987.

Celik, T. 2009. "Multiscale Change Detection in Multitemporal Satellite Images." IEEE Geoscience and Remote Sensing Letters 6 (4): 820-824. doi:10.1109/LGRS.2009.2026188.

Celik, T. 2010, May. "A Bayesian Approach to Unsupervised Multiscale Change Detection in Synthetic Aperture Radar Images." Signalling Processing 90 (5): 1471-1485. doi:10.1016/j. sigpro.2009.10.018.

Chatelain, F., J.-Y. Tourneret, J. Inglada, and A. Ferrari. 2007, July. "Bivariate Gamma Distributions for Image Registration and Change Detection." IEEE Transactions on Image Processing 16 (7): 1796-1806. doi:10.1109/TIP.2007.896651. 
Cui, S., and M. Datcu. 2012, August. "Statistical Wavelet Subband Modeling for Multi-Temporal SAR Change Detection." IEEE Journal of Selected Topics in Applied Earth Observations and Remote Sensing 5 (4): 1095-1109. doi:10.1109/JSTARS.2012.2200655.

Cui, S., G. Schwarz, and M. Datcu. 2016, March. "A Benchmark Evaluation of Similarity Measures for Multitemporal SAR Image Change Detection." IEEE Journal of Selected Topics in Applied Earth Observations and Remote Sensing 9 (3): 1101-1118. doi:10.1109/JSTARS.2015.2486038.

Dumitru, C. O., S. Cui, G. Schwarz, and M. Datcu. 2015, April. "Information Con-tent of Very-HighResolution SAR Images: Semantics, Geospatial Context, and On-tologies." IEEE Journal of Selected Topics in Applied Earth Observations and Remote Sensing 8 (4): 1635-1650. doi:10.1109/ JSTARS.2014.2363595.

Grigorescu, S. E., N. Petkov, and P. Kruizinga. 2002, October. "Comparison of Texture Features Based on Gabor Filters." IEEE Transactions on Image Processing 11 (10): 1160-1167. doi:10.1109/ TIP.2002.804262.

Gueguen, L., and M. Datcu. 2009. "Mixed Information Measure: Application to Change Detection in Earth Observation." In Proceedings of the 5th International Workshop on the Analysis of Multitemporal Remote Sensing Images. Groton, CT: Multi-temp. http://www.proceedings.com/13014. html

Inglada, J., and G. Mercier. 2007, May. "A New Statistical Similarity Measure for Change Detection in Multitemporal SAR Images and Its Extension to Multiscale Change Anal-ysis." IEEE Transactions on Geoscience and Remote Sensing 45 (5): 1432-1445. doi:10.1109/ TGRS.2007.893568.

Kandaswamy, U., D. A. Adjeroh, and M. C. Lee. 2005, September. "Efficient Texture Analysis of SAR Imagery." IEEE Transactions on Geoscience and Remote Sensing 43 (9): 2075-2083. doi:10.1109/ TGRS.2005.852768.

Li, H.-C., T. Celik, N. Longbotham, and W. J. Emery. 2015, December. "Gabor Feature Based Unsupervised Change Detection of Multitemporal SAR Images Based on Two-Level Clustering." IEEE Geoscience and Remote Sensing Letters 12 (12): 2458-2462. doi:10.1109/LGRS.2015.2484220.

Manjunath, B. S., and W. Y. Ma. 1996. "Texture Features for Browsing and Retrieval of Image Data." IEEE Transactions on Pattern Analysis and Machine Intelligence 18 (8): 837-842. doi:10.1109/34.531803.

Mercier, G., S. Derrode, W. Pieczynski, J.-M. Nicolas, A. Joannic-Chardin, and J. Inglada. 2006. "Copula-based Stochastic Kernels for Abrupt Change Detection." In Proceedings of the IEEE International Geoscience Remote Sensing Symposium (IGARSS), 204-207. Denver, CO: Institute of Electrical and Electronics Engineers (IEEE).

Mercier, G., and J. Inglada. 2008. "Change Detection with Misregistration Errors and HetErogeneous Data through the Orfeo Toolbox." Technical report, Institut TELECOM, TELECOM Bretagne.

Rignot, E. J. M., and J. J. Van Zyl. 1993, July. "Change Detection Techniques for ERS-1 SAR Data." IEEE Transactions on Geoscience and Remote Sensing 31 (4): 896-906. doi:10.1109/36.239913.

Slaney, M., and M. Casey (2008). Locality-Sensitive Hashing for Finding Nearest Neighbors [Lecture Notes]. IEEE Signal Processing Magazine 25, 128-131. doi:10.1109/MSP.2007.914237.

Wald, I., and V. Havran. 2006. On Building Fast Kd-Trees for Ray Tracing, and on Doing that in O (Nlogn). In 2006 IEEE Symposium on Interactive Ray Tracing, edited by I. Wald and S. G. Parker, 61-69. IEEE. doi:10.1109/RT.2006.280216

Wang, Q., S. R. Kulkarni, and S. Verdu. 2009, May. "Divergence Estimation for Multidi-Mensional Densities via K -Nearest-Neighbor Distances." IEEE Transactions on Infor-mation Theory 55 (5): 2392-2405. doi:10.1109/TIT.2009.2016060.

Wikipedia. 2016. TanDEM-X. Accessed 23 April 2016. https://en.wikipedia.org/wiki/TanDEM-X

Winter, A., H. Maitre, N. Cambou, and E. Legrand. 1997, April. "Entropy and Multiscale Analysis: A New Feature Extraction Algorithm for Aerial Images." In Proceedings of the IEEE International Conference on Acoustics, Speech, and Signal Processing (ICASSP), Vol. 4, 2765-2768. Munich: IEEE Computer Society.

Xiong, B., J. M. Chen, and G. Kuang. 2012. "A Change Detection Measure Based on A Likelihood Ratio and Statistical Properties of Sar Intensity Images." Remote Sensing Letters 3 (3): 267-275. doi:10.1080/01431161.2011.572093. 